\title{
Correlation of Gastric Aspirate Polymorphs And Acute Phase Reactants (M-Esr, Crp And Band Cell Count) With Blood Culture In Early onset Neonatal Sepsis - A Tertiary Care Study
}

\author{
Dr.Chandramohan Chekkali ${ }^{1}$, Dr.Narahari Bapanpally ${ }^{2}$, \\ Dr.Ajay Mohan Varahala ${ }^{3}$ \\ ${ }^{1,2,3}$ Assistant Professor of Pediatrics, Niloufer Hospital, Hyderabad, India \\ Corresponding Author: Dr.B.Narahari,
}

\begin{abstract}
Introduction: Mortality due to early onset neonatal sepsis is much higher than late onset sepsis. Clinical manifestations of the early onset sepsis are non specific. Delay in treatment can lead to complications and death of the neonates.

Objective: To detect the sensitivity, specificity and the positive predictive values of the gastric aspirate polymorphs and the acute phase reactants in relation with the blood cultures of the neonates with early onset sepsis.

Materials And Methods: Study was conducted at a tertiary care center. 50 neonates were included in the study. Investigations like blood culture, CRP, micro ESR, band cell count and gastric aspirate polymorphs count was done. All the observations were recorded. The sensitivity, specificity, positive predictive value and the negative predictive values were calculated based on the observations.

Results: In the present it was seen that $41.02 \%$ of the cases of neonatal sepsis with GA polymorphs have a sensitivity of $72.72 \%$, and a positive predictive value of $41.02 \%$ with blood culture. In regard to neonatal sepsis, $m$-ESR was found to be correlated with a sensitivity and specificity of $54.54 \%$ and $67.85 \%$ respectively. In cases of neonatal sepsis, sensitivity and specificity of band forms and blood culture is $78.57 \%$ and $75 \%$ respectively.

Conclusion: The present study concludes that CRP and Band forms are useful in the early detection of early neonatal sepsis as both have high sensitivity, specificity and positive predictive values.

Keywords: Band forms, blood cultures, CRP, early onset neonatal sepsis, gastric aspirate polymorphs, micro ESR, positive predictive values, sensitivity, and specificity.
\end{abstract}

\section{Introduction}

Sepsis is a major cause of morbidity and mortality in the neonatal period ${ }^{1}$. Neonatal sepsis is classified depending on the hours of presentation into ${ }^{2} 1$ ) Early onset: within first 72 hours of life. 2) Late onset: occurring after 72 hours of life. Early onset neonatal sepsis is often due to organisms present in the maternal vaginal flora. ${ }^{2}$ Mortality in this condition is much higher than in late onset sepsis. Early diagnosis and prompt antimicrobial therapy is necessary for managing this condition. Infection in early neonatal period is one of the important factors responsible for high perinatal mortality and neonatal morbidity in developing countries. Early manifestations of neonatal septicemia are vague and nonspecific ${ }^{2}$. Delay in the institution of antimicrobial therapy is fraught with dangers of several complications and increase in mortality. Isolation of the infecting organism from blood provides the definitive diagnosis and is considered as the 'gold standard'. However, this culture procedure takes at least 48 hours to confirm the diagnosis. Therapy cannot wait this long in a critically sick neonate. On the other hand the indiscriminate overuse of antibiotics on the basis of clinical suspicion alone is hazardous for any neonatal unit, because it will lead to emergence of resistance. Hence, to rationalize antimicrobial therapy in neonatal sepsis, certain indirect early markers of neonatal infections have been identified.

There are various predisposing factors that lead to an increased neonatal susceptibility to infection. These include $^{1}$ :a) VLBW( $<1500$ gms $)$ or Preterm baby, b) Febrile illness in the mother during or within two weeks of delivery, c) Foul smelling liquor, d) PROM $>12 \mathrm{hrs}$, e) Frequent vaginal examinations $(>$ three), f) Prolonged and difficult labor with instrumentation, g) Birth asphyxia and difficult resuscitation, h) Pathological evidences of funisitis or presence of polymorphs in the gastric aspirate. These risk factors operating during perinatal period are precise determinants of the ultimate neonatal outcome.

Markers for infection which are used for diagnosing are as follows ${ }^{1}$ : 
I) Direct Markers Of Neonatal Infection ${ }^{1}$ : Cultures of blood, cerebrospinal fluid (CSF) and urine are taken before initiating antibiotic therapy.

II) Indirect Markers Of Neonatal Infection ${ }^{1}$ : A number of early indirect markers of infection can be used as a mean of suspecting diagnosis of neonatal septicemia:

i) Leukocyte counts: ratios and morphology

ii) Micro erythrocyte sedimentation rate (m-ESR)

iii) Acute phase proteins: A number of acute phase proteins serve to indicate the presence of infection in the neonate.

a) Those increasing with inflammation are: i) CRP, ii) Alpha 1 acid glycoprotein, iii) Haptoglobin, iv) Alpha 1 antitrypsin, v) Fibrinogen

b) Those decreasing with inflammation are: i) Pre-albumin, ii) Transferrin

Role of gastric aspirate in the diagnosis of early neonatal sepsis ${ }^{2}$ :

Several screening procedures have been in use like cultures of the cord blood, peripheral blood, ear canal debris, and histology of the umbilical placenta, whole mount of amnion and frozen sections of umbilical cord for evidence of vasculitis. Recently, microscopic appearances of fluid from the ear canal and gastric aspirate have been used as evidence of bacterial sepsis acquired in utero. Blanc ${ }^{3}$ detected evidence of inflammation from a smear of the fetal surface of the placenta and presence of leucocytes in the gastric aspirate of the fetus. Bernirschke ${ }^{4}$ introduced the technique of microscopic examination on rapid frozen sections of the umbilical cord and correlated umbilical wall inflammation with infection. Oliver ${ }^{5}$ has suggested a relationship between the presence of polymorph nuclear cells in the gastric aspirate and the possibility of subsequent infection of the newborn. This method of using criteria of cells in the gastric fluid of an infant appears to be the simplest and one of the most readily carried out investigation without the requirement of specialized personnel and equipment. It can be done in a side laboratory. Thus, demonstration of bacteria and inflammatory cells in the gastric aspirate on the first day of life (within an hour of life) may reflect maternal amnionitis. Present study is conducted to correlate the gastric aspirate polymorphs and acute phase reactants (m-ESR, CRP and BAND Cell Count) with blood culture in early neonatal sepsis.

\section{Aims And Objectives}

a) To detect sensitivity; specificity \& positive predictive value of Gastric Aspirate (GA) Polymorphs and Acute Phase Reactants (m-ESR, CRP and Band Cell Count) to diagnose early onset neonatal sepsis (i.e., with in 72 hrs of life).

b) To correlate between duration of rupture of membranes \& other maternal risk factors (fever within 2 weeks prior to delivery; foul smelling liquor) for positive sepsis screen with gastric aspirate polymorphs and Acute Phase Reactants.

\section{Materials And Methods}

The study was conducted in Department of Paediatrics, Niloufer hospital, which is a teaching institute and an allied hospital under Osmania Medical College, Hyderabad. The hospital is a tertiary care referral centre for both obstetric and pediatric patients. The hospital has a Level III NICU for neonatal emergencies. Fifty neonates with signs of early onset sepsis or with maternal risk factors for sepsis were included in the study.

\section{The inclusion criteria for this study:}

a. Neonates with symptoms \& signs of sepsis.

b. Preterm less than 36 weeks or low birth weight less than $2500 \mathrm{gms}$.

c. Maternal fever in preceding 2 weeks.

d. Foul smelling liquor.

e. Premature rupture of membranes. (More than 12hours).

f. More than 3 vaginal examinations in labour.

g. Prolonged or difficult delivery with instrumentation.

The exclusion criteria for this study:

1. Full term healthy neonates with no maternal risk factors.

2. Inborn errors of metabolism, birth asphyxia \& Meconium Aspiration Syndrome.

3. Transient metabolic states i.e. hypoglycaemia.

4. Physiological hypothermia.

After selecting the neonate for study, their details were recorded as per fixed proforma containing identification data, detailed history,findings of clinical examination \& investigation results. 
The following investigations were done for all the neonates selected for the study:-

a. Gastric aspirate for polymorphs.

b. Haemoglobin, TC, DC with Band Cell Count.

c. Peripheral smear for toxic granules.

d. Micro ESR.

e. CRP.

f. Blood culture.

g. CSF analysis (when indicated).

h. Urine, pus (from superficial focus) Culture [when indicated].

i. Babies were subjected to radiological investigations (when indicated).

\section{Procedure of gastric aspirate cytology in neonates:}

Immediately after baby was born with risk factors, NG tube was inserted and gastric fluid was collected and sent to the pathology department. There smear was done by standard technique by Leishman's stain and 200 cells were counted. The percentage of polymorph nuclear cells was calculated. The percentage of more than 5 cells per HPF was considered as positive for infection.

Interpretation of results: The results of the above sepsis screen panel were interpreted in the following manner.

a. GA showing $>5$ polymorphs/high power field (HPF) is considered positive.

b. CRP was done by a semi quantitative method and a value of $>1 \mathrm{mg} / \mathrm{dL}$ has been considered positive.

c. Band cell count of more than $20 \%$ of total leukocyte is considered as positive

d. Micro-Erythrocyte Sedimentation Rate (micro-ESR) value greater than $15 \mathrm{~mm}$ after 1 hour at any age has been considered positive.

e. Blood culture done in every case of suspected sepsis and the results compared to that of the screening tests.

Blood Culture and antibiotic sensitivity was done using appropriate media and disk diffusion method respectively.

The following investigations were done for all the mothers selected for the study:-

a. High vaginal swab during labour.

b. Total \& differential counts.

\section{Iv. Observations \& results}

Out of the 56 cases of suspected neonatal sepsis that were selected for the study, six cases were excluded from the study as they manifested features of hypoglycemia which was confirmed by glucose estimation $(<40.0 \mathrm{mg} / \mathrm{dL})$. Hence, only 50 cases were included in the study $(\mathrm{N}=50)$.

Table I Sex Wise Distribution Of The Neonates With Sepsis ( $\mathrm{N}=50)$

\begin{tabular}{|l|l|}
\hline Sex & $\mathrm{N}=50(\%)$ \\
\hline Male & $28(56 \%)$ \\
\hline Female & $22(44 \%)$ \\
\hline
\end{tabular}

In present study, males (56\%) are more than females (44\%) with a ratio of 1.27: 1

Table Ii Positivity Of Ga Polymorphs, Acute Phase Reactants (M-Esr, Crp, Band Cell Count) And Blood Culture In Neonatal Sepsis ( $\mathrm{N}=50)$

\begin{tabular}{|c|c|c|c|}
\hline Serial No & Criteria & Positive (\%) & Negative (\%) \\
\hline 1 & Ga For Polymorphs & $39(78 \%)$ & $11(22 \%)$ \\
\hline 2 & Micro Esr & $21(42 \%)$ & $29(58 \%)$ \\
\hline 3 & Crp & $22(44 \%)$ & $28(56 \%)$ \\
\hline 4 & Band Cell Count & $24(48 \%)$ & $26(52 \%)$ \\
\hline 5 & Blood Culture & $22(44 \%)$ & $28(66 \%)$ \\
\hline
\end{tabular}

Gastric aspirate for Polymorphs were observed in a maximum (78\%) number of cases, and in a minimum $(42 \%)$ in the 'increased micro ESR group. The gold standard of diagnosis, i.e. blood culture, was positive in $44 \%$ of the cases.

Table III correlation Between Sexes Of Neonates With Positive Blood Cultures ( $\mathrm{N}=50$ )

\begin{tabular}{|c|c|c|}
\hline Sex & No Of Cases=N $(\%)$ & Blood Culture +Ve=N (\%) \\
\hline Male & $28(56 \%)$ & $12(54.54 \%)$ \\
\hline Female & $22(44 \%)$ & $10(45.45 \%)$ \\
\hline
\end{tabular}


In the present study, blood cultures were positive more in male neonates (54.54\%) than in females $(45.45 \%)$.

Table IV Comparision Of Ga Polymorphs And Blood Culture In Neonatal Sepsis (N=50)

\begin{tabular}{|c|c|c|}
\hline \multirow{2}{*}{ Ga Polymorphs } & \multicolumn{2}{|c|}{ Blood Culture } \\
\cline { 2 - 3 } & Positive & Negative \\
\hline Positive (39) & 16 (True Positive) & 23 (False Positive) \\
\hline Negative (11) & 6 (False Negative) & 5 (True Negative) \\
\hline Total N=50 & 22 & 28 \\
\hline
\end{tabular}

Sensitivity $=16 / 22 \times 100=72.72 \%$,

Specificity $=5 / 28 \times 100=17.85 \%$,

Positive predictive value $=16 / 39 \times 100=41.02 \%$,

Negative predictive value $=5 / 11 \times 100=45.45 \%$

From the above it is observed that in cases of neonatal sepsis, $41.02 \%$ of positive GA polymorphs were also blood culture positive. It could also be seen that $41.02 \%$ of the cases of neonatal sepsis with GA polymorphs have a sensitivity of $72.72 \%$, and a positive predictive value of $41.02 \%$ with blood culture.

Table v

Comparision of m-esr and blood culture in neonatal sepsis $(\mathrm{n}=50)$

\begin{tabular}{|c|c|c|}
\hline \multirow{2}{*}{ M-Esr } & \multicolumn{2}{|c|}{ Blood Culture } \\
\cline { 2 - 3 } & POSITIVE & NEGATIVE \\
\hline Positive 21 & 12 (True Positive) & (False Positive) \\
\hline Negative 29 & 10 (False Negative) & 19 (True Negative) \\
\hline Total N=50 & 22 & 28 \\
\hline
\end{tabular}

Sensitivity $=12 / 22 \times 100=54.54 \%$,

Specificity $=19 / 28 \times 100=67.85 \%$,

Positive predictive value $=12 / 21 \times 100=57.14 \%$,

Negative predictive value $=19 / 29 \times 100=65.51 \%$

With regards to neonatal sepsis, m-ESR \& Blood culture was found to be correlated with a sensitivity and specificity of $54.54 \%$ and $67.85 \%$ respectively.

Table Vi Comparison Of Crp And Blood Culture (N=50)

\begin{tabular}{|c|c|c|}
\hline \multirow{2}{*}{ Crp } & \multicolumn{2}{|c|}{ Blood Culture } \\
\cline { 2 - 3 } & Positive & Negative \\
\hline Positive (22) & 17 (True Positive) & 5 (False Positive) \\
\hline Negative (28) & 5 (False Negative) & 23 (True Negative) \\
\hline Total N=50 & 22 & 28 \\
\hline
\end{tabular}

Sensitivity $=17 / 22 \times 100=77.27 \%$,

Specificity $=23 / 28 \times 100=82.14 \%$,

Positive predictive value $(\mathrm{PPV})=17 / 22 \times 100=77.27 \%$,

Negative predictive value $($ NPV) $=23 / 28 \times 100=82.14 \%$

In cases of neonatal sepsis, CRP and blood culture was found to be correlated with a sensitivity and specificity of $77.27 \%$ and $82.14 \%$ respectively, with PPV of $77.27 \%$ and an NPV 0 f $82.14 \%$

Table Vii Comparison Of Band Forms And Blood Cultures (N=50)

\begin{tabular}{|c|c|c|}
\hline \multirow{2}{*}{ Band Forms } & \multicolumn{2}{|c|}{ Blood Culture } \\
\cline { 2 - 3 } & Positive & Negative \\
\hline Positive (24) & 18 (True Positive) & 6 (False Positive) \\
\hline Negative (26) & 4 (False Negative) & 22 (True Negative) \\
\hline Total N=50 & 22 & 28 \\
\hline
\end{tabular}

Sensitivity $=18 / 22 \times 100=81.81 \%$,

Specificity $=22 / 28 \times 100=78.57 \%$,

Positive predictive value $(\mathrm{PPV})=18 / 24 \times 100=75.0 \%$,

Negative predictive value $(\mathrm{NPV})=22 / 26 \times 100=84.61 \%$

In cases of neonatal sepsis, sensitivity and specificity of band forms and blood culture is $81.81 \%$ and $78.57 \%$ respectively, with PPV of $75 \%$ and an NPV of $84.61 \%$. 
Table Viii Correlation Of Ga Polymorphs \& Acute Phase Reactants With Maternal Risk Factors (N=50)

\begin{tabular}{|c|c|c|c|c|}
\hline Serial No & Criteria & Prom $(\mathrm{N}=7)(\%)$ & $\begin{array}{c}\text { Perinatal } \\
\text { Fever }(\mathrm{N}=1) \\
(\%)\end{array}$ & $\begin{array}{c}\text { Foul Smelling } \\
\text { Liquor (N=1) }(\%)\end{array}$ \\
\hline 1 & Ga Poly Morphs & $5(71.42 \%)$ & 0 & $1(100 \%)$ \\
\hline 2 & M-Esr & $2(28.7 \%)$ & 0 & 0 \\
\hline 3 & Band Cell & $1(14.28 \%)$ & 0 & 0 \\
\hline 4 & Crp & $1(14.28 \%)$ & 0 & $1(100 \%)$ \\
\hline 5 & Blood Culture & $2(28.7 \%)$ & 0 & 0 \\
\hline
\end{tabular}

Neonates born to mothers of PROM had GA polymorphs in $71.42 \%$, whereas blood culture positive was seen in only $28.7 \%$.

\section{Discussion}

Neonatal septicemia is a leading cause of mortality and morbidity in neonates. Early diagnosis will help the clinicians to decide on the usage of proper antibiotics which will help in reducing the morbidity and mortality. A positive blood culture is the only definitive method of confirming a case of septicemia. Culture and sensitivity tests require a minimum period of 48 hours which is a precious time in making decisions in the treatment of sepsis in newborns. Rapid Diagnosis by doing band cell count and CRP estimations give a reasonable degree of accuracy in diagnosis of neonatal septicemia. High index of clinical suspicion and early investigation helps clinicians in diagnosis and appropriate treatment. Abuse of antibiotics in case of conditions which simulate septicemia can thus be avoided.

Table Ix Comparative Study Of Blood Culture Positivity In Neonatal Sepsis

\begin{tabular}{|c|c|c|}
\hline Serial No & Author & Percentage Of Positive Blood Culture \\
\hline 1 & Guha Et Al $^{6}$ & $40 \%$ \\
\hline 2 & Namdeo Et Al & $50.08 \%$ \\
\hline 3 & Rao P.S Et Al & $40 \%$ \\
\hline 4 & Present Study & $44 \%$ \\
\hline
\end{tabular}

In the present study, blood culture positivity is similar to studies by others. ${ }^{6,7}$ In_the present study blood culture positivity is found to be $44.0 \%$ The success in achieving a positive culture among other studies: Guha etal ${ }^{6}$ reported $40.0 \%$, Namdeo etal ${ }^{7}$ reported $50.0 \%$ and Rao PS etal ${ }^{8}$ reported $40.0 \%$. However negative cultures do not rule out sepsis. Guha etal ${ }^{6}, 1978$ reported 7 cases with negative blood culture but with fatal outcome and postmortem evidence of infection.

Table X Comparitive Study Of Ga Polymorphs In Early Neonatal Sepsis

\begin{tabular}{|c|c|c|}
\hline Serial No & Author & Positivity (\%) \\
\hline 1 & Je Hg Jeoung Etal $^{9}$ & $75 \%$ \\
\hline 2 & Chandana Etal $^{10}$ & $44 \%$ \\
\hline 3 & Present Study & $78 \%$ \\
\hline
\end{tabular}

In the present study positivity of GA polymorphs $(78.0 \%)$ is comparable with Je HG Jeoung. YM etal ${ }^{9}$. Others ${ }^{10}$ reported fewer figures due to the fact that the study included a less number of cases with maternal risk factors.

Table Xi Comparitive Study Of Micro Esr In Early Neonatal Sepsis

\begin{tabular}{|c|c|c|c|}
\hline Serial No & Author & Sensitivity (\%) & Specificity (\%) \\
\hline 1 & $\begin{array}{c}\text { Anitha Sharma } \\
\text { Etal }^{11}\end{array}$ & $60 \%$ & $62.5 \%$ \\
\hline 2 & P.K.Mishra Etal & & \\
\hline 3 & Walliullah Etal & & \\
\hline 4 & Present Study & $63 \%$ & $62 \%$ \\
\hline
\end{tabular}

The present study is similar to studies done by others ${ }^{11,13}$. P.K. Mishra ${ }^{12}$ observed the sensitivity and specificity to be higher than the present study as they considered more than $8 \mathrm{~mm}$ in the first one hour as an increased $\mathrm{m}$-ESR value.

Table XII Comparative Study Of Sensitivity\&Specificity Of Band Forms In Early Neonatal Sepsis

\begin{tabular}{|c|c|c|c|}
\hline Serial No & Author & Sensitivity (\%) & Specificity (\%) \\
\hline 1 & Gerdes Js Etal $^{14}$ & $65 \%$ & $73 \%$ \\
\hline 2 & S.N.Parida Etal $^{15}$ & $84 \%$ & $66 \%$ \\
\hline 3 & Present Study & $81.81 \%$ & $78.57 \%$ \\
\hline
\end{tabular}


The present study is similar with other studies ${ }^{14,15}$. In the past, the changes in the white blood cell parameters among neonates were regarded least useful for the diagnosis of sepsis as these values were thought to be too erratic. Gerdes JS etal ${ }^{14}$ studied these changes more precisely in healthy and diseased neonates and established its usefulness as a supportive test for the diagnosis of neonatal sepsis. In the present study emphasis was placed on band forms because they constitute the first line of defense. More over neutrophils comprise the major cell population during immediate neonatal period. Present study correlate closely with the findings of other workers ${ }^{14,}{ }^{15}$. Though the neonates are at a disadvantage due to poorly developed immune response, nonetheless they do respond to an infective insult as an older child. During an acute infection neutrophils are rapidly released from the neutrophils storage compartment to circulation, which manifest as band forms and provide potent cells for migration to infected tissues. In S.N.Parida etal ${ }^{15}$ study, 7 babies from both the groups had leucopenia and $71.0 \%$ of them died. This was due to either due to marrow failure or increase sequestration. This finding is remarkable when viewed in context that total neutrophils in both the groups did not differ significantly. Present study observations are in agreement with those of S.N.Parida etal ${ }^{15}$, who have cautioned that one should not be deceived by neutrophils count alone without noting alteration in the ratio of mature and immature neutrophils. Increased band count as a sensitive indicator of sepsis, as observed in present study has also been documented by other workers. It is apparent that while investigating septic neonates, increase in percentage of band forms in peripheral blood smear as an acceptable predictive value of $75 \%$ as was observed in the present study. In neonates with fulminant infections, leucopenia may occur and band count is often unaltered.

Table Xiii Comparitive Study Of Sensitivity\&Specificity Of Crp In Neonatal Sepsis

\begin{tabular}{|c|c|c|c|}
\hline Serial No & Author & Sensitivity (\%) & Specificity (\%) \\
\hline 1 & I.M.Singh Etal $^{16}$ & $80 \%$ & $91 \%$ \\
\hline 2 & P.Kite Etal $^{17}$ & $61.8 \%$ & $81.2 \%$ \\
\hline 3 & Berger Etal $^{18}$ & $75 \%$ & $86 \%$ \\
\hline 4 & Present Study & $77.27 \%$ & $82.14 \%$ \\
\hline
\end{tabular}

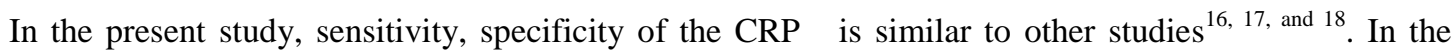
present study the blood culture was positive in only $44 \%$ cases. The low positivity of blood culture underlines the need of other tests in diagnosing neonatal septicemia. Out of the various individual tests for rapid diagnosis of neonatal septicemia, in proved sepsis group CRP was the one with maximum sensitivity (77.2\%) and specificity of a $(82.14 \%)$. Other workers have also observed similar high sensitivity and specificity with CRP, P. Kite etal ${ }^{17}(61.80 \%, 81.20 \%)$ and Berger etal ${ }^{18}(75 \%, 86 \%)$ respectively. Of the rapid diagnostic tests, CRP was found to be most useful when taken singly. Its elevation and returning to normal levels once the infection is controlled occurs in a matter of a few hours. Kite etal ${ }^{17}$ have reported elevated CRP levels in $80 \%$ of cases of neonatal sepsis.

Table Xiv Comparitive Study Of Maternal Risk Factors With Blood Culture In Neonatal Sepsis

\begin{tabular}{|c|c|c|c|c|c|c|c|}
\hline S No & Author & Prom & $\begin{array}{c}\text { Culture } \\
\text { Proven }\end{array}$ & $\begin{array}{c}\text { Perinatal } \\
\text { Fever }\end{array}$ & $\begin{array}{c}\text { Culture } \\
\text { Proven }\end{array}$ & $\begin{array}{c}\text { Foul } \\
\text { Smelling } \\
\text { Liquor }\end{array}$ & $\begin{array}{c}\text { Culture } \\
\text { Proven }\end{array}$ \\
\hline 1 & $\begin{array}{c}\text { Betty Chacko } \\
\text { Etal }^{19}\end{array}$ & 21 & $6(5.7 \%)$ & 1 & 0 & 3 & 0 \\
\hline 2 & $\begin{array}{c}\text { Sangamitra } \\
\text { Etal }^{20}\end{array}$ & 10 & $1(1 \%)$ & 1 & $1(4 \%)$ & 4 & $1(4 \%)$ \\
\hline 3 & Present Study & 7 & $2(28.5 \%)$ & 1 & 0 & 1 & 0 \\
\hline
\end{tabular}

In the present study, culture proven cases are more $(28.5 \%)$ in PROM as compared to other studies ${ }^{19,20}$. It could be due to intra partum antibiotics administration in other studies.

\section{Conclusions}

Fifty cases of neonatal sepsis were studied to correlate the gastric aspirate polymorphs and acute phase reactants (mESR, CRP and BAND CELL COUNT) with blood culture in early neonatal sepsis. In present study, males $(56 \%)$ are more than females (44\%) with a ratio of 1.27: 1. Gastric aspirate for Polymorphs were observed in a maximum (78\%) number of cases, and in a minimum (42\%) in the increased micro ESR group. The gold standard of diagnosis, i.e. blood culture, was positive in $44 \%$ of the cases. In the present study, blood cultures were positive more in male neonates $(54.54 \%)$ than in females $(45.45 \%)$. It is observed that in cases of neonatal sepsis, $41.02 \%$ of positive GA polymorphs were also blood culture positive. It could also be seen that $41.02 \%$ of the cases of neonatal sepsis with GA polymorphs have a sensitivity of $72.72 \%$, and a positive predictive value of $41.02 \%$ with blood culture. In regard to neonatal sepsis, m-ESR was found to be correlated with a sensitivity and specificity of $54.54 \%$ and $67.85 \%$ respectively. In cases of neonatal sepsis, sensitivity and 
specificity of band forms and blood culture is $78.57 \%$ and $75 \%$ respectively and PPV was $81.82 \%$ and NPV was $84.61 \%$. Neonates born to mothers of PROM had GA polymorphs in $71.42 \%$, whereas blood culture positive was seen in $28.7 \%$.

CRP can be used for screening of early neonatal sepsis as its sensitivity, specificity and positive predictive value is high, i.e. $72.2 \%, 82.14 \%$ and $77.2 \%$ respectively. Band Forms is one of the useful indicators for diagnosing neonatal Sepsis as its sensitivity, specificity and positive predictive values are high, i.e. $81.81 \%$, $78.57 \%, 75.0 \%$ respectively. Premature rupture of membranes (PROM) is a common maternal risk factor for early onset neonatal sepsis, as blood culture is positive in $28.7 \%$._Sensitivity, specificity and positive predictive value of GA Polymorphs are 72.72\%, 17.85\%, 41.0\% and micro ESR 54.54\%, 67.85\%, 57.14\% respectively. In conclusion, CRP and Band forms are more useful than GA polymorphs and micro ESR in screening of early neonatal sepsis.

\section{References}

[1]. Klein JO, Remington JS, infectious disases of the fetus and newborn infant,5th ed. Philadelphia, WB Saunders 2001, pp943-998

[2]. Singh M; Care of the New born $6^{\text {th }}$ Ed. 2004 sagar publications New delhi pp209-218

[3]. Blanc WA. Amniotic infection syndrome. Clin Obstet Gynecol 1959, 2: 705.

[4]. Benirschke K., Driscoll SG. The pathology of the human placenta. Berlin Heidelberg and New York (quoted in the manual of neonatal care.) $2^{\text {nd }}$ edn. Igakushain pp108. )

[5]. Oliver TX. Jr. The physical diagnosis of newly born. Report of $46^{\text {th }}$ Ross conference on pediatric research Columbia. $1964 \mathrm{pp} 47$.

[6]. Guha DK ,Jaspal. D, Krishna Das MS. Outcome of neonatal septicemia Clinical and Bacterological profile .Indian Pediatr 1978, 15: 423-27.

[7]. Namdeo UK, Singh HR, RAjput VJ, Kushwana. Hematological indices for early diagnosis of neonatal septicemia. Indian Pediatr 1985, 22: 287-292

[8]. Rao P.S. Baligam, Shivananda PG. Bacteriology of neonatal septicemia in rural referral hospital in south india.J. Trop.Paed.1993:39(4): 230-233.

[9]. Je HG Jeoung YM. Diagnostic value of various screening tests in neonatal sepsis . Korean J pediatr 2006. 46(11): 1167-1173.

[10]. Chandna AM, Nagaraj Rao, Srinivas M, Shyamala S. Rapid diagnostic tests in neonatal septicemia. Indian J Pediatr 1988, 55:94753.

[11]. Anitha sharma, Krishna kutty CV,Sabharwal U,Rathee Sushila,Harash Mohan.Evaluation of sepsis screen or diagnosis of neonatal septicemia.Indian J Pediatr 1993,60:559-563.

[12]. Mishra PK, Sharma S,Mallik GK,Agarwal SK. Simple tests for early detection of neonatal infections. Indian Pediatr 1983,20:44750 .

[13]. Walliullah Sm, Islam, Siddika M. Hassain MA, Chowdhury AK. Micro ESR in early onset sepsis. Mymensingh med J.2009: 18(1):56-61.

[14]. Gerdes JS, Polin R. Early diagnosis and treatment of neonatal sepsis. Indian J Pediatr 1998. 65: 63- 78.

[15]. Parida, S.N., Verma, I.C. \& Singh, M. Blood leucocyte changes for the early diagnosis of neonatal sepsis Indian J Pediatr (September,1982) Vol.49: 653-657.

[16]. Singh I M, Narang A, Bhakoo ON. Evaluation of sepsis screen in the diagnosis of neonatal sepsis. Indian Pediatr 1987,24:39-40

[17]. Kite P, Millar MR. Comparision of five tests in the diagnosis of neonatal bacteremia.Archive of disease in childhood.1983, 63: 639643.

[18]. Berger C. Comparision of C-Reactive Protein and White blood cell count with differential in neonates at risk for septicemia; Eur J Pediatr 1995,154: 138-144.

[19]. Betty Chacko, Inderpreet Sohi. Early onset neonatatal sepsis. Indian J Pediatr 2005, 72:23-26.

[20]. Sanghamithra Datta, Jaswinder K.Oberai, TD. Chugh. Laboratory diagnosis of Neonatal Sepsis.J.Neon 2006, 20:16-23.

[21]. Srinivas murki,Sudhakar,Ravi Shankar, Ananth reddy,Laxmi. Rectal Sole Temperature Difference: A Marker of Neonatal Septicemia. J Neon 2006; 20: pp45-48

[22]. Karen M. Pupopola. Bacterial and fungal infection in manual of neonatal care. $5^{\text {th }}$ edn. Lippincott Williams and Willkins 2004 . 287313.

[23]. Agarwal RC, Ansari Z, Ahmed SH, Dutta AK, Rajendra Kumar. Gastric aspirate examination for early detection of neonatal septicemia. Indian pediatr. 1980, 17: 458-461.

[24]. Paul VK, Singh M. Diagnosis and treatment of neonatal sepsis. Indian pediatr. 1986, 23:1023-1035. 